Supporting Information

\title{
Phase and Dispersion Stability of Silver Nanocolloids for Nanoparticle-Chemisorption Printing
}

Yuya Hirakawa, Keisuke Aoshima, *Shunto Arai, "Tatsuo Hasegawa.

${ }^{*}$ Corresponding Authors

E-mail: arai@ap.t.u-tokyo.ac.jp (S. Arai), t-hasegawa@ap.t.u-tokyo.ac.jp (T. Hasegawa).

Department of Applied Physics, The University of Tokyo, Tokyo 113-8656, Japan

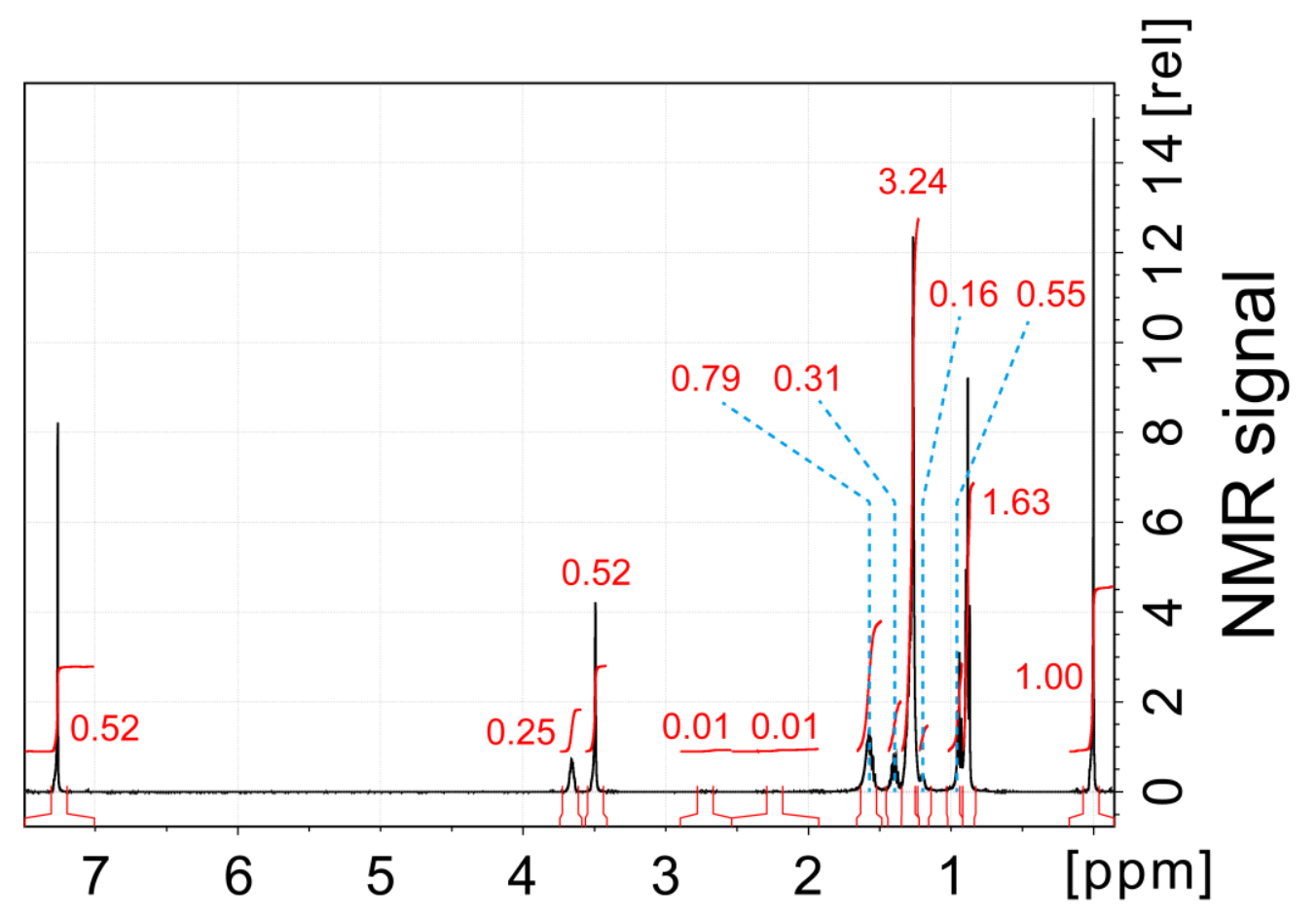

Figure S1. A typical NMR spectrum of the AgNCs. The peaks around 1.26, 3.49, and 3.65 ppm correspond to octane, butanol, and methanol, respectively. The figure in red under each peak is the integrated value of the peak. Volume fraction of each solvents is calculated by integrating the area under the corresponding peak. 


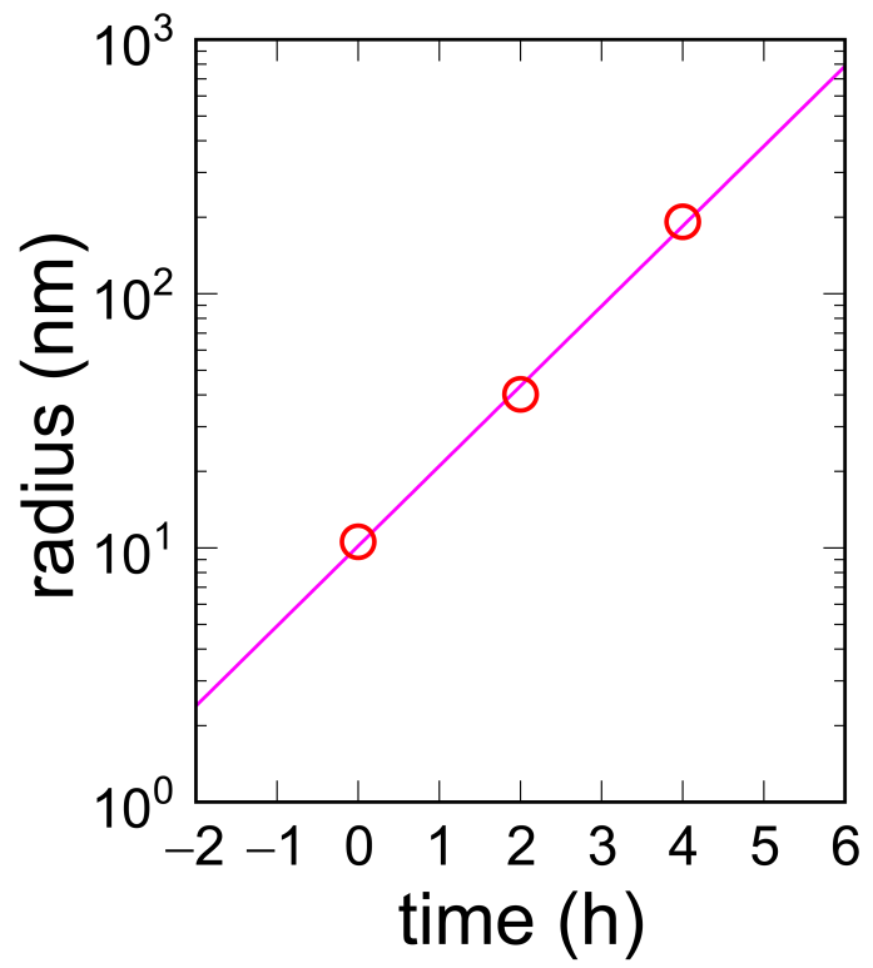

Figure S2. Growth of average size of colloidal clusters. To identify the growth rate, the obtained results were fitted with an exponential function: $f(t)=r_{0} \exp (t / \tau)$. We obtained the value of $r_{0}$ and $\tau$ as the fitting parameters, and determined as $10.2 \mathrm{~nm}$ and 1.4 hour, respectively. 

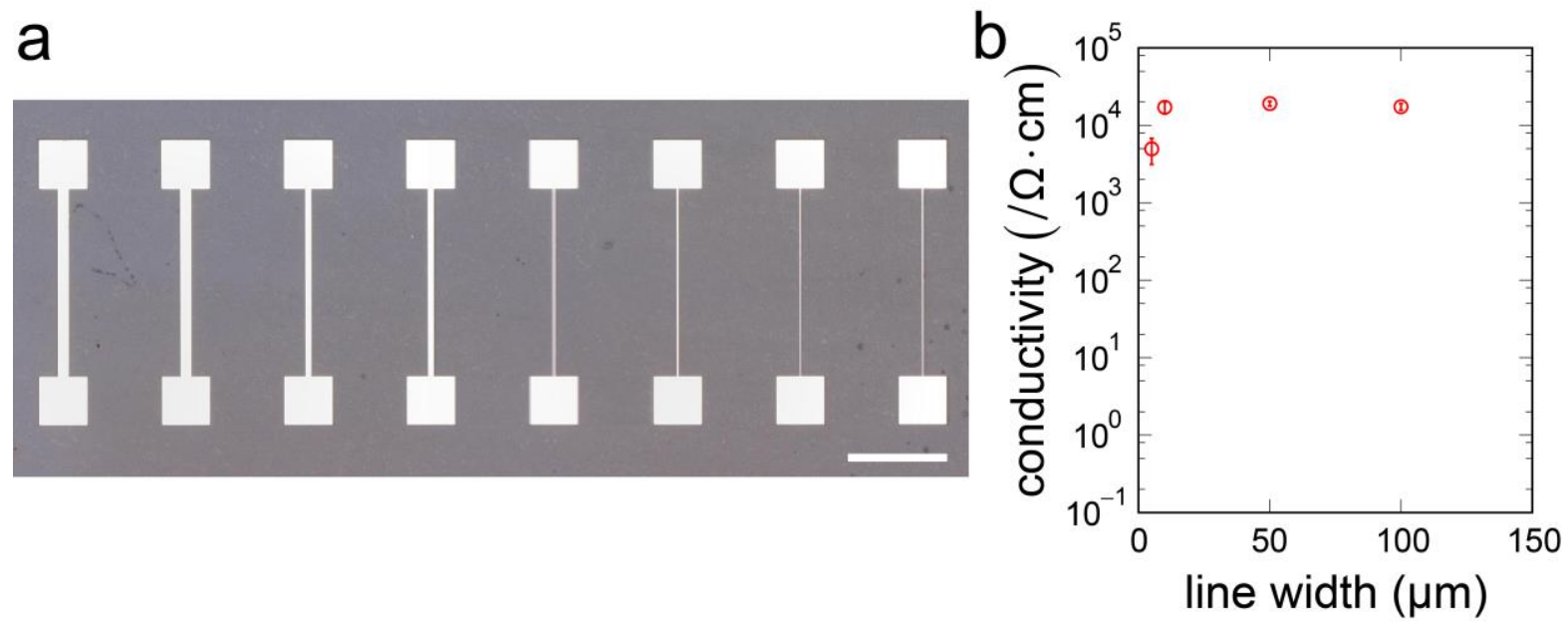

Figure S3. Conductivity measurements of printed electrodes with low-temperature thermal treatment. The electrodes are fabricated using AgNCs containing 12.3\% methanol. (a) Optical micrograph of the printed silver electrodes fabricated by the SuPR-NaP technique. These electrodes are treated with $50{ }^{\circ} \mathrm{C}$ for 2 hours after drying at room temperature for 2 days. The scale bar is $1 \mathrm{~mm}$. (b) Conductivity of fabricated electrodes. The conductivity is measured for 5, 10, 50 and $100 \mu$ m-line width. 8 samples are measured and the results are averaged for each line width. 